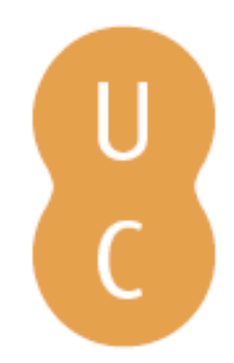

\title{
pommalina
}

\section{Immanenz und Selbstbezug der künstlerischen Form: skizze zum Verhältnis von Hölderlin und Spinoza}

\author{
Autor(es): $\quad$ Büttner, Stefan \\ Publicado por: Imprensa da Universidade de Coimbra \\ URL \\ persistente: URI:http://hdl.handle.net/10316.2/31621 \\ DOI: $\quad$ DOI:http://dx.doi.org/10.14195/978-989-26-0205-9_5 \\ Accessed : $\quad$ 26-Apr-2023 11:56:23
}

A navegação consulta e descarregamento dos títulos inseridos nas Bibliotecas Digitais UC Digitalis, UC Pombalina e UC Impactum, pressupõem a aceitação plena e sem reservas dos Termos e Condições de Uso destas Bibliotecas Digitais, disponíveis em https://digitalis.uc.pt/pt-pt/termos.

Conforme exposto nos referidos Termos e Condições de Uso, o descarregamento de títulos de acesso restrito requer uma licença válida de autorização devendo o utilizador aceder ao(s) documento(s) a partir de um endereço de IP da instituição detentora da supramencionada licença.

Ao utilizador é apenas permitido o descarregamento para uso pessoal, pelo que o emprego do(s) título(s) descarregado(s) para outro fim, designadamente comercial, carece de autorização do respetivo autor ou editor da obra.

Na medida em que todas as obras da UC Digitalis se encontram protegidas pelo Código do Direito de Autor e Direitos Conexos e demais legislação aplicável, toda a cópia, parcial ou total, deste documento, nos casos em que é legalmente admitida, deverá conter ou fazer-se acompanhar por este aviso.

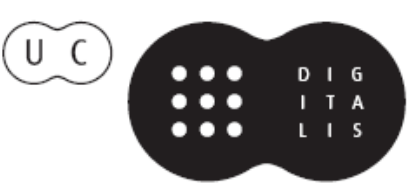


Edmundo Balsemão Pires

Burkhard Nonnenmacher

Stefan Büttner-von Stülpnagel

Editors

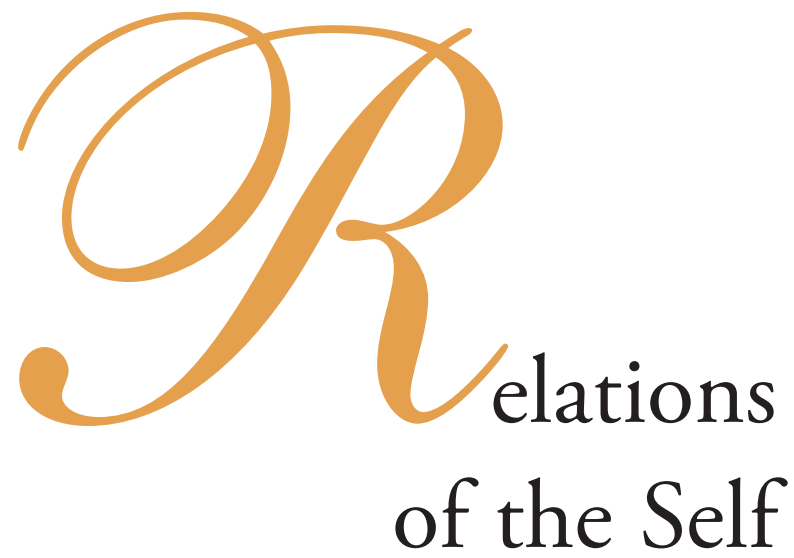




\section{Stefan Büttner}

Potsdam

\section{IMMANENZ UND SELBSTBEZUG DER KÜNSTLERISCHEN FORM. SKIZZE ZUM VERHÄLTNIS VON HÖLDERLIN UND SPINOZA ${ }^{1}$}

Es ist nicht zu bestreiten, dass Spinoza für Hölderlin ein wichtiger philoso-phischer Bezugspunkt war.

Nicht zuletzt darum, weil die Umkehrung des Spinozabildes im Weimarer Neuspinozismus der 80er Jahre des 18. Jahrhunderts in Hölderlins intellektuell prägende Lebensphase fällt.

Spinoza wird nach 100jähriger Verfemung als ein Atheist par excellence schlagartig vom Verdammten zum Verehrten, vom Verfluchten zum Gesegneten.

Dieser Umschwung kann zu Recht als „kulturrevolutionäres Ereignis“ bezeichnet werden.

Dies konnte keinen Intellektuellen unberührt lassen. Zumal einen jungen Dichter wie Hölderlin - einen wachen Zeitgenossen der Französischen Revolution - , der in der Revolution den realen Aufgang der Kantischen Freiheit sah, und der zugleich durch einen chiliastischen Pietismus geprägt war auf das nahe Reich Gottes. Eine Vision, säkular und religiös zugleich.

Nur wenige Jahre nach dem kulturellen und ein Jahr nach dem realen revolu-tionären Umschwung datiert Hölderlins erste Beschäftigung mit Spinoza. Hölderlin lagen dabei drei Texte zu und über Spinoza vor, von denen zwei direkt, einer indirekt dessen Lehre von der All-Einheit, von der ,Gott-Natur“ zum Thema hatten: Jacobis „Über die Lehre des Spinoza in Briefen an Herrn Moses Mendelssohn“, Herders „Gott. Einige Gespräche“ und Heinses „Ardinghello und die glückseeligen Inseln“.

Jacobi hat Hölderlin intensiv studiert und exzerpiert, Herder hat er zur Kenntnis genommen, mit Heinse war er freundschaftlich verbunden - ihm hat er eines seiner schönsten Gedichte, die Elegie „Brot und Wein“, gewidmet.

Dies ist bekannt. Schwieriger ist zu zeigen, wie Hölderlin Spinoza rezipiert hat und in welcher Form spinozanische Grundgedanken in die Grundlegung seiner Philosophie, die in ästhetisch-poetischer Absicht von Hölderlin entworfen wird, eingehen.

Und noch schwieriger erscheint es, in Hölderlins poetischem Verfahren, dem von ihm so genannten „Wechsel der Töne“, einer Lehre für die Verfertigung von Sprachkunstwerken, Grundkonzepte Spinozas identifizieren zu wollen. Ist doch Spinoza derjenige unter Hölderlins Leitsternen, zu denen vor allem Platon und Pindar, Schiller und Kant zu zählen sind, der sich am wenigsten, ja überhaupt nicht mit Kunst und Kunsttheorie beschäftigt hat.

\footnotetext{
${ }^{1}$ Published as well in: M. Bollacher, T. Kisser u. M. Walther (Hrsg.): Spinoza in Literatur un Ästhetik, Würzburg 2009.
} 
Ich möchte in meinen Ausführungen jedoch entgegen diesem ersten Eindruck und in Ergänzung der bisherigen Forschung Spinozas Konzeption als entscheidenden Referenzpunkt für Hölderlins Dichtung und Dichtungstheorie aufzeigen, indem ich Spinozas Philosophie als diejenige Hintergrundphilosophie herausarbeite, durch deren Transformation Hölderlins eigener philosophischer Ansatz und seine damit verbundene Dichtungstheorie erst verständlich wird. ${ }^{2}$ Hölderlins Sprachkunstwerke als Einheit von sprachlich-ästhetischer Valenz und intellektueller Form werden so in den Kontext einer von Spinoza sich herleitenden Ausdrucks-philosophie des Absoluten gestellt.

Um diesen Nachweis führen zu können, werde ich mich im ersten Teil meiner Ausführungen zunächst nur mit Hölderlins späten Hymnen - Sprachkunstwerken aus den Jahren 1799 bis 1803 - und der sie fundierenden Dichtungstheorie beschäftigen.

Hölderlins späte Hymnen - so meine erste These - präsentieren das All-Eine auf solche Weise, dass sie die Welt als Ausdruck des All-Einen zur Darstellung bringen. Die Hymnen präsentieren das All-Eine, das Absolute auf adäquate Weise. Diese ästhetische Präsentation des Absoluten ist zentrales Thema und Kernaufgabe der Hölderlinschen Hymnen. Aus diesem Grund ist Hölderlins Poesie als absolute Poesie zu verstehen.

Im zweiten Teil werde ich drei spinozanische Theoriestücke als die philoso-phischen Rahmenbedingungen nennen, die Hölderlins späte Hymnen ermöglichen. Denn adäquate Ausdrucksform des All-Einen kann ein Sprachkunstwerk nur sein, wenn das Verhältnis zwischen dem All-Einen und seiner weltlichen und sprachlichen Darstellung mit dem von Spinoza erfundenen Ausdruckskonzept gefaßt wird, das die Immanenz des All-Einen mit dessen Absolutheit zusammen zu denken vermag. Kein anderes philosophisches Konzept als das des Spinoza erlaubt es in dieser Zeit - so meine zweite These - , von einer adäquaten Darstellung des All-Einen durch das Endliche zu sprechen. Denn nur wenn die Welt insgesamt als Ausdruck Gottes verstanden wird, kann sich in der endlichen Welt das Absolute als absolutes Zeichen zeigen.

In diesem Zusammenhang wird sich auch erweisen, welche Bedeutung Spinozas absolute Immanenzphilosophie für Hölderlins Gottesbegriff hatte. So sollte mit Hilfe eines neuen, eben spinozistischen ,Begriffs von der Gottheit' sowohl die bloß auf Endlichkeiten fixierte Nützlichkeitstheorie vermieden, als auch die christliche Transzendenzkonzeption abgewendet werden, ohne die Rede von Gott aufgeben zu müssen.

Im dritten Teil werde ich zeigen, welche Folgen Spinozas Ausdrucks-philosophie für Hölderlins künstlerische Form hat. Ich werde andeuten, inwiefern Hölderlins Konzeption der Töne als eine Konsequenz der spinozanischen Auffassung des Modus verstanden werden kann. Hier werde ich dann die These des ersten Teils begründen, warum jedes Hölderlinsche Sprachkunstwerk als adäquate Darstellung des All-Einen zu verstehen ist. Meine daran anschließende dritte These lautet: Hölderlins Dichtung ist - am präzisesten - als Selbstdarstellung und als Selbstaussage des All-Einen zu verstehen, so dass jedes Sprachkunstwerk Folge des All-Einen und zugleich dessen Realität ist.

Der Gesang ist - wie es in einer späten Hymne heißt - eine „Sage der Liebe“ (Am Quell der Donau), dergestalt, dass der Gesang sich der göttlichen Liebe verdankt und zugleich diese Liebe ist, der er sich verdankt. Spinozas „amor Dei intellectualis“ in poetischer Form.

\footnotetext{
${ }^{2}$ Für Hölderlins Hyperion hat dies prägnant gezeigt Wegenast (1990), S. 126 ff.
} 


\section{Hölderlins absolute Poesie: die späten Hymnen als adäquate Ausdrucksform des All- Einen}

Die späten Sprachkunstwerke - also die späten Hymnen, die Elegien und Nachtgesänge - und die sie begründende Dichtungstheorie sind Zentrum und Zielpunkt des Hölderlinschen Werks. Sie sind für Hölderlin die Lösung der philosophischen Grundfrage, die sich ihm seit 1794 in steigender Intensität und Komplexität stellt.

Ich werde daher im Folgenden in bewusster Beschränkung nur auf Hölderlins späte Hymnen eingehen - also auf die Gruppe von Werken, die von der Feiertagshymne bis zu Mnemosyne reichen. Dabei werde ich eine prosaische Darstellung der Hölderlinschen Hymnen und vor allem eine kurze Zusammenfassung der Ausdruckskonzeption als dem zentralen Thema dieser Hymnen geben. Ganz bewusst werde ich nicht beim frühen Hölderlin und beim viel traktierten Fragment Urteil und Sein beginnen, das Auftakt des Hölderlinschen Philosophierens und nicht dessen ausgebildete oder gar durchgearbeitete Konzeption ist.

In seinen späten Hymnen entwirft Hölderlin eine Geschichtsphilosophie, die die Einheit der Geschichte in der Abfolge verschiedener Kulturen zum Inhalt hat. Die Einheit der Geschichte bezeichnet er mit dem poetischen terminus technicus als „Wort“. Dieses „Wort“ ist an verschiedenen historisch-geographischen Orten in besonderer Ausprägung aufgetreten. Diese besonderen Stationen und insbesondere die sie jeweils bestimmenden Prinzipien sind „Gipfel der Zeit“ (Patmos: 2,1: 165). Diese Stationen sind zunächst die Welt der jüdischen Patriarchen und Propheten, dann die Welt der griechischen Athleten und Dichter und dann die Welt der römischen Kultur. Jede dieser Welten ist eine bestimmte Darstellung des „Worts“, des „Geistes“, wie Hölderlin auch sagen kann, dessen Gang in Asien beginnt und über Griechenland und Rom in den Bereich von Donau und Rhein führt, den Hölderlin zuweilen Hesperien nennt, wo der Geist die Jetzt-Zeit und die geographische Umwelt des Dichters erreicht hat.

Im Bereich von Donau und Rhein angekommen erscheint, das Wort, die „menschenbildende Stimme“ (Am Quell der Donau: 2,1: 126) als ein göttliches Ereignis, das die Menschen - all ihren technischen Fähigkeiten zum Trotz - im Alltag nicht aufnehmen können. Denn dieser Geist übersteigt ihre normalen kognitiven und emotionalen Fähigkeiten. ${ }^{3}$

„Doch einige wachten“ - so heißt es bei Hölderlin (Am Quell der Donau: 2,1: 12127). Sie sind in der Lage den Geist wahrzunehmen und aufzunehmen. Und diese wenigen werden in der poetischen Erinnerung an die früheren Orte des Geistes versetzt - eben nach Ionien, an den Kaukasus, nach Syrien - an Orte, an denen einige Zwiesprache mit dem Göttlichen zu halten und in ein Wechselverhältnis mit dem Absoluten einzutreten in der Lage waren. Diese Rückkehr zu den Stationen des Geistes wird als quasi objektives Geschehen in den späten Hymnen geschildert.

Dann erfolgt in den Hymnen ganz generell eine Wendung des Gedankens: Die Einheit der Geschichte wird eigens benannt - und zwar auf poetisch-mythische Weise.

In der Hymne Am Quell der Donau spricht sich dies so aus: „Aber wenn ihr, Und diß ist zu sagen, Ihr Alten all, nicht sagtet woher? Wir nennen Dich, heiliggenöthiget, nennen

${ }^{3}$ In der poetischen Terminologie der Hymne Am Quell der Donau heißt das: Die Menschen schlafen angesichts dieser göttlichen Gabe ein, ihr Augenlicht ist erloschen. 
Natur! dich wir und neu, wie dem Bad entsteigt, Dir alles Göttlichgeborene“ (Am Quell der Donau: 2,1: 126). Das „Woher“ ist die Frage nach der Einheit der Geschichte. Wir, das sind Hölderlins Zeitgenossen. "Heiliggenöthiget" das ist eine Aussage mit absoluter Notwendigkeit. Und „Natur“ ist die Antwort auf die Frage nach der Einheit der Geschichte.

„Natur“ ist nämlich der poetisch-mythische terminus technicus für diese Einheit. Wobei „Natur“ für das übergreifend Allgemeine steht, das in zwei Sphären ausgedrückt wird. In der Sphäre des Menschen, von Hölderlin auch die Sphäre der Kunst (im Sinn von ,Techne') genannt, und in der Sphäre der Götter, von Hölderlin auch als Sphäre der Natur im engeren Sinne gefaßt. An dieses übergreifende Allgemeine, die All-Eine Natur will das Sprachkunstwerk so erinnern, dass die Natur selbst präsent wird im Gedicht.

Wenn aufgrund dieser Erinnerung an das übergreifend Allgemeine die poetisch-mythische Präsentation der Natur, des All-Einen, „Gottes“ in der Hymne geleistet wird, dann erfüllt die Hymne für die Zeitgenossen ihre belebende Funktion. Sie bereitet den Aufbau einer neuen von Geist erfüllten Welt vor, die durch die Rezipienten der Hölderlinschen Hymnen, durch Hölderlins Zeitgenossen, dann ins Werk gesetzt werden müßte.

Um diese hier sehr knapp skizzierte poetische Konstruktion der Geschichte, die in den späten Hymnen zwar in verschiedenen Akzentuierungen, aber in übereinstimmender Grundstruktur durchgängig ist, deutlicher zu machen, will ich auf Hölderlins philosophische Begründung zurückgreifen, die er im Fragment Das Werden im Vergehen gibt.

In Das Werden im Vergehen spricht Hölderlin von der jeweiligen Kultur - z. B. der der Griechen - als einer „besonderen Welt“. Diese besondere Welt ist dadurch gekennzeichnet, dass sie geworden ist und wieder vergeht. Der Grund für Entstehen und Vergehen dieser besonderen Welt ist, dass die besondere Welt nur ein bestimmter, nur ein besonderer Ausdruck der „Welt aller Welten“ ist (WiV, 4,1: 282).

Die Welt aller Welten kann als solche gar nicht zum Ausdruck kommen. Sie kann nur in der Abfolge der besonderen Welt, genauer im Moment des Untergangs einer besonderen Welt und des Entstehens einer neuen besonderen Welt zur Darstellung, zur Erscheinung kommen. „Denn die Welt aller Welten, das Alles in Allen, welches immer ist, stellt sich nur in aller Zeit oder im Untergange oder im Moment, oder genetischer im Werden des Moments und Anfang von Zeit und Welt dar, und dieser Untergang und Anfang ist wie die Sprache Zeichen, Darstellung eines lebendigen, aber besonderen Ganzen“. (WiV, 4,1: 282).

Die Welt aller Welten ist immer, sie ist ewig. Als ewige Welt aller Welten kann sie sich aber nur in aller Zeit, also in einer endlosen Abfolge der besonderen Welten darstellen.

Zugleich garantiert die Welt aller Welt, das Alles in Allen den Übergang der besonderen Welten, deren Werden und Vergehen. Präziser gesagt: Sie ist die Substanz in allen Übergängen.

Es ist nämlich das Absolute, das sich im Umschlag der Welten, im Werden und Vergehen, als solches und damit adäquat darstellt. Denn das Absolute, die Welt aller Welten stellt sich im Moment des Umschlags als Besonderheit und als Totalität zugleich dar. Ein etwas längeres Zitat von Hölderlin formuliert dies ganz präzise:

Denn wie könnte die Auflösung empfunden werden ohne Vereinigung, wenn also das Bestehende in seiner Auflösung empfunden werden soll und empfunden wird, so muß dabei das Unerschöpfte und Unerschöpfliche, der Beziehungen und Kräfte, und jene, die Auflösung, mehr durch diese empfunden werden, als umgekehrt (...) Aber das Mögliche, welches in die 
Wirklichkeit tritt, indem die Wirklichkeit sich auflöst, dies wirkt, und es bewirkt sowohl die Empfindung der Auflösung als die Erinnerung des Aufgelösten." (WiV: 4,1: 282)

Hölderlin denkt sich die Welt aller Welten als die Unerschöpflichkeit der Beziehungen und Kräfte. Wenn nun eine besondere Welt untergeht, dann tritt im Moment des Untergangs die Unerschöpflichkeit der Welt aller Welten hervor. Und zwar so, dass diejenigen Kräfte der Natur, die nicht in den Bau dieser besonderen nun untergehenden Welt eingegangen sind, sich als auflösendes Prinzip zeigen, - als unbestimmte Möglichkeiten, die in diesem Untergang, der dadurch ein Übergang ist, erscheinen und zugleich zur sprachlichen Darstellung drängen.

Das heißt: Die Unerschöpflichkeit der Welt aller Welten bewirkt sowohl die Empfindung der Auflösung als auch die Erinnerung des Aufgelösten. Die Welt aller Welten zeigt sich in der Sphäre des Bewußtseins, des Geistes als die Möglichkeit des Neuen. Sie wirkt im Geist, auch wenn das Neue noch nicht sichtbar und das Alte bereits verschwunden ist. Der Geist, die Sphäre des Mentalen ist also in besonderer Weise mit der Welt aller Welten verbunden.

Unter diesem bis jetzt skizzierten geschichtsphilosophischen Fokus wird ganz klar, was Hölderlins poetische Konstruktion in der Hymne leisten will. Sie will nicht die historischen Ereignisse schildern, sie auch nicht in ihrer Abfolge vorführen - im Sinne einer empirischen oder geschichtswissenschaftlichen Darstellung der historischen Ereignisse und Epochen. Die Hymne gibt vielmehr eine ästhetisch-philosophische Konstruktion dieser Ereignisse, die dadurch in dieser Konstruktion erst als solche Ereignisse real werden.

Dies kann das Sprachkunstwerk nur leisten, wenn es das empirische, das geschichtliche Ereignis zugleich und ineins als intellektuelles, als transempirisches Ereignis fasst; wenn es Einheit von Empirisch-Faktischem und Transzendental-Gesetzlichem ist, wenn es im Hölderlinschen Sinne „mythisch“ bzw. eine Mythe ist.

Eine solche Mythe im Sinne Hölderlins ist die „Donau“. Denn die Donau ist einerseits empirisch-faktisch gesehen ein Fluß, ein beobachtbares Ding in der Welt, der der geographischen Beschreibung zugänglich ist. Andererseits steht die Donau hinsichtlich der rechtlichen oder intellektuellen Verhältnisse für bestimmte Lebensverhältnisse, Gewohnheiten, Sitten, Rechte und Normen. Die besondere Welt, die sich in der Landschaft entlang der Donau gebildet hat, unterscheidet sich spezifisch von der Welt der Griechen hinsichtlich Religion, Politik und Kultur.

Zugleich weist der Fluß, die Donau, in ihrem Verlauf nach Osten, nach Asien, nach Griechenland. Die Lebensverhältnisse unserer Welt weisen so auf die Welt der Griechen, auf Asien hin. Aber so, dass der empirische Verlauf des Flusses nun - in der Perspektive absoluter Poesie - zur Realität des Geschichtsverlaufs wird. Der Fluß, die Donau, ist die Geschichte vom Gang des Wortes aus Osten: Denn die Donau geht nach Osten und geht zugleich so nach Osten, dass es - aufgrund ihrer langsamen Fließgeschwindigkeit - so scheint, als fließe sie rückwärts und käme wirklich aus Osten.

Die Donau als Sprachkunstwerk wird damit die empirisch-intellektuelle und so die poetisch-mythische Realität, die den Gang des Wortes aus Osten Realität werden läßt. Im Fokus der Dichtung ist die Donau absolutes Zeichen für das „Wort aus Osten“. Sie ist Einheit von Prinzip und Faktum. Ein Naturphänomen als solches ist Geschichte.

Was hat Hölderlins Konzept der Mythe nun mit Spinoza zu tun? 


\section{Spinozas Ausdruckskonzeption als Rahmenbedingung der absoluten Poesie}

Zur Beantwortung dieser Frage werden im zweiten Teil die Grundgedanken der spinozanischen Ausdrucksphilosophie dargestellt und drei Entsprechungen zwischen Hölderlin und Spinoza aufgezeigt. Im Sinne meiner zweiten These heißt dies, dass nur Spinozas Ausdruckskonzeption eine absolute Poesie im Hölderlinschen Sinne möglich gemacht hat.

Wir hatten gesehen, dass der empirisch-faktische Verlauf der Donau als Zeichen des Geistes der Donau gedeutet wird, also als Zeichen unseres intellektuellen Verhältnisses zu den griechischen und asiatischen Stationen des Geistes. Der empirische Fluß wird durch das Hölderlinsche Sprachkunstwerk zur „Mythe“ und damit zur Darstellung des Übergangs von der alten Welt zur neuen Welt. Die Donau ist das Prinzip für die neue Welt.

Das dieses Prinzip darstellende und damit das die Mythe konstituierende Sprachkunstwerk wird in einem präzisen Sinn zum adäquaten Ausdruck des All-Einen. Denn der Übergang von einer besonderen Welt zur nächsten besonderen Welt kann nur gelingen, wenn jede besondere Welt als Ausdruck der Welt aller Welten verstanden wird und zwar so, dass in diesen empirischen, besonderen Welten immer der Ausdruckscharakter mitgedacht wird. Jede besondere Welt muss in ihrer Zweidimensionalität erkannt werden: Einerseits besondere Welt zu sein, andererseits Ausdruck des All-Einen, Ausdruck der Welt aller Welten.

Diese Erkenntnis findet nach Hölderlin beim Untergang einer alten Welt und dem Werden einer neuen Welt statt und findet in der Mythe des Dichters ihre Realität.

Um diese poetische Theorie formulieren zu können, bedarf es - erstens - eines neuen Begriffs des Absoluten, zweitens einer neuen Theorie vom Verhältnis zwischen Absolutem und Endlichem und damit einem neuen Verhältnis der endlichen Dinge untereinander und drittens einer neuen Form der Erkenntnis des Absoluten. Alle drei Innovationen fand Hölderlin bei Spinoza:

a. Damit sind wir bei der ersten Entsprechung zwischen Hölderlin und Spinoza, die die Substanz bzw. das Absolute betrifft: Der, Welt aller Welten' bei Hölderlin entspricht die Substanz bei Spinoza - der Substanz, die durch die Attribute auf eine qualitativ-bestimmte Weise ausgedrückt wird, die ihrerseits wieder durch die Modi auf eine gewisse und bestimmte Weise ausgedrückt werden. Damit ist bei Spinoza jeder Modus eine bestimmte Weise die Substanz zu zeigen, aber so, dass in der Substanz bzw. in den Attributen nichts Qualitatives verborgen bleibt, sondern so, dass die Modi die Substanz bzw. die Attribute adäquat ausdrücken, ohne doch selbst absolut oder unendlich zu sein. Sie sind als Modi Einschränkungen, Einteilungen der Unendlichkeit der Attribute, Modifikationen der Attribute und darum endlich, aber von ihnen nicht qualitativ unterschieden.

Die Funktion der Substanz ist es, die Modi und deren Verhältnis möglich zu machen. Sie ist die absolute Identität, die nicht ist, sondern nur gezeigt werden kann. ${ }^{4}$ Und zwar gezeigt durch die endlichen Modi. Diese Zweidimensionalität ist das Spezifikum der Ausdruckskonzeption. Ihr folgt Hölderlin ohne Einschränkung. ${ }^{5}$

\footnotetext{
${ }^{4}$ Vgl. Büttner (2009)

${ }^{5}$ Denn sie ermöglicht ihm - wie vielen seiner Zeitgenossen nach dem von Jacobi entfesselten Streit um Spinoza Frömmigkeit, Weltfrömmigkeit zu entwickeln, ohne den orthodox-transzendenten Gottesbegriff annehmen zu müssen.
} 
Denn diese spezifisch spinozanische Zweidimensionalität der Welt, der Immanenz des Absoluten, zeigt sich - in Hölderlins Geschichtskonzeption - im Moment des Untergangs und des Entstehens, des Vergehens und Werdens, weil in jedem Systemuntergang die Totalität aller Umweltbeziehungen zur Erscheinung kommt. Zugleich ist damit das Wissen von der Absolutheit des All-Einen verbunden. Wenn ich also den Untergang als Übergang weiß, dann habe ich den Zusammenhang, genauer die Identität mit der untergegangenen Welt konstituiert.

b) Ich komme nun zur zweiten Entsprechung zwischen Hölderlin und Spinoza, die die endlichen Modi betrifft. Die Entsprechung betrifft die Art und Weise, wie die Substanz, die Welt aller Welten durch den jeweiligen Modus, durch die besondere Welt und deren Werden und Vergehen ausgedrückt wird und welche Auswirkungen dies für die Verfassung des Endlichen und dessen Präsentation des Absoluten hat. Hier gilt bei Spinoza und Hölderlin: Das Endliche ist Manifestation des Absoluten in adäquater, wenn auch begrenzter Form. Die begrenzte Form präsentiert das Absolute, ohne dass durch die Begrenzung die Qualität des Absoluten, in unserem Fall, die Qualität der Attribute berührt wird. Zugleich und ineins damit ist die begrenzte Form nicht das Absolute selbst.

Das Absolute kommt in jedem Modus zur Erscheinung, ohne selbst Erscheinung zu sein. Es handelt sich hier um ein absolutes Darstellungsverhältnis, bei dem keines der Relate gestrichen werden kann.

Mit dem Grundbegriff der Ontologie, dem „ens“, ist dieses Verhältnis überhaupt nicht denkbar. Hölderlin verabschiedet mit Spinoza Metaphysik und Erkenntnis-theorie des „ens“, die Philosophie des Seins und des Seienden, also das, was klassischerweise Ontologie genannt wird. Aus diesem Grund ist die Rede vom „Sein“ bei Hölderlin als transzendentem Grund verfehlt, denn sie beruht auf der falschen Voraussetzung, als sei die Substanz ein Seiendes jenseits der Modi. Denn Hölderlins Grund ist - eben so wie derjenige Spinozas ein offenbarer Grund, denn er wird durch die Modi gezeigt, ohne mit den Modi identisch zu sein. Ausdruck eben. Das heißt: Die endlichen Dinge drücken die Substanz aus, aber so, dass sie die Selbigkeit der Substanz in unterschiedlichen Formen, in den absoluten Formen Denken und Ausdehnung, ausdrücken. Diese Ausdrücke sind dann die einzelnen Körper und Gedanken.

Hölderlin eignet sich Spinozas Theorie vom Verhältnis der Substanz zu den beiden die Welt konstituierenden absoluten Formen Denken und Ausdehnung nun so an, dass er die Unterscheidung der Attribute als Grundunterscheidung in der Welt, durch die das Absolute ausgedrückt wird, als Unterscheidung des Absoluten selbst einführt. Denn die Ausdrucksfigur zwischen Absolutem und Welt verlangt, dass die Welt, damit sie sich vom Absoluten unterscheidet, als Unterscheidung (als Ur-teilung) eintritt. Diese Unterscheidung formuliert Hölderlin als Unterscheidung von Natur und Kunst. Natur und Kunst sind die Formen, in der das Absolute auftritt. Natur ist dabei diejenige Seite der Unterscheidung, die für die überschüssigen Kräfte steht, die nicht in die jeweilige historisch-kulturelle Welt eingehen. Kunst steht - als die andere Seite der ursprünglichen Unterscheidung - als Terminus für die kulturelle Welt.

Im Rahmen dieser Unterscheidung von Natur und Kunst treten dann die Naturphänomene wie Wasser, Luft, Erde und Feuer, Flüsse und Gebirge, Pflanzen und Tiere als modal 
unterschiedene in der Natur auf. Als nur modal von ihrer Sphäre, vom Attribut Natur unterschiedene sind die Naturdinge zu Zeichen und Präsentanten, zu Ausdrücken des Absoluten geworden, die das Absolute in ihrer Relationalität darstellen.

Ein Gedanke, den Hölderlin bei Heinse, Herder und bei Jacobi finden konnte. Mit Jacobis Formulierung gesagt: „Die einzelnen Dinge setzen sich, gegenseitig, einander voraus und beziehen sich aufeinander, so dass eines davon ohne alle die übrigen, und alle die übrigen ohne dieses eine, weder seyn noch gedacht werden können; das heißt sie machen ein unzertrenntliches Ganzes aus" (Jacobi: Spinozabüchlein 1. Auflage: 4, 202).

Eine korrespondierende Stelle aus Hölderlins Roman Hyperion sei hier angeführt - auf dessen letzter Seite heißt es: „Ihr Quellen der Erd! Ihr Blumen! Und ihr Wälder, und ihr Adler und du brüderliches Licht! Wie alt und neu ist unsere Liebe! - Frei sind wir, gleichen uns nicht ängstig von außen; wie sollte nicht wechseln die Weise des Lebens? Wir lieben den Aether doch all und innigst im Innern gleichen wir uns" (Hyperion: 3, 160). Die Formen des Ausdrucks sind verschieden, ihr Wesen ist identisch.

c) Ich komme zur dritten Entsprechung zwischen Hölderlin und Spinoza, die das Verhältnis von Substanz und unendlichem Modus betrifft: Die konstitutive Bezogenheit von Verstand („Geist“) und All-Einem.

Wir hatten bei der Darstellung der Hölderlinschen Geschichtsphilosophie gesehen, dass der Übergang von der alten Welt in die neue Welt nur als ein gewußter Übergang überhaupt ein Übergang ist. Nicht gewußt und ästhetisch präsentiert, handelt es sich nur um eine Abfolge von Welten, deren eine, die alte, vergangen ist, wenn die neue entstanden ist. Auf die Einsicht des Übergangs jedoch kommt es Hölderlin an, denn nur diese Einsicht kann die Welt aller Welten präsentieren. Der Übergang ist also konstitutiv mit dem Bereich des Bewußtseins verbunden, was Hölderlin überzeugt sein läßt, seine eigene Position als Zusammenführung von Spinoza und Kant zu verstehen. Bei Spinoza selbst besteht eine unablösbare Ausdrucksbeziehung ebenfalls - und zwar zwischen Substanz und unendlichem Verstand. Im unendlichen Verstand wird repräsentiert, was in allen Attributen ist.

Poetisch stellt sich diese Einheit, dieses Ausdrucksverhältnis von Verstand und Substanz bei Hölderlin so dar, dass das Verhältnis von Menschen und Göttern so gedacht wird, dass den Menschen der mitfühlende Part zugesprochen wird. „Denn weil die Seeligsten nichts fühlen von selbst, Muß wohl, wenn solches zu sagen Erlaubt ist, in der Götter Nahmen / Teilnehmend fühlen ein Andrer, / Den brauchen sie (...)“. (Der Rhein: 2,1: 145)

Die Götter sind zwar nicht die Substanz, aber sie sind Ausdruck der All-Einen Natur und bilden eine eigene Sphäre, die durch den Menschen gefühlt wird. Bei Spinoza ist es der unendliche Verstand, von dem der menschliche Geist ein Teil ist, der alle anderen Attribute repräsentiert. So versteht sich die Substanz im unendlichen Verstand, denn als sie selbst denkt sie nicht. Sie denkt sich im Attribut des Denkens als unendliche Modifikation. Analog zeigt sich Hölderlins Natur in den Göttern, der Sphäre der Natur im engeren Sinne, und denkt und fühlt sich in den Menschen, der Sphäre der Kunst. ${ }^{6}$

In diesem Zustand bin ich, wie Hölderlin sagt, einig „mit allem, was lebt“ (Über den Unterschied der Dichtarten, 4,1: 267). Diese Einigkeit mit allem, was lebt, identifiziert er

\footnotetext{
${ }^{6}$ Vgl. Büttner (1988/89)
} 
ganz zu Recht mit der „intellektualen Anschauung“. Denn die intellektuale Anschauung ist die Einigkeit mit allem, was lebt, weil sie der Mitvollzug dieses All-Einen Lebens ist. Und von dieser Einigkeit mit allem, was lebt, heißt es dann im Fragment Über den Unterschied der Dichtarten: Sie ist eine, „die zwar von dem beschränkteren Gemüte nicht gefühlt, die in seinen höchsten Bestrebungen nur geahndet, aber vom Geiste erkannt werden kann" (Über den Unterschied der Dichtarten, 4,1: 267 f.). ${ }^{7}$

Dass dieser Geist sich dann ästhetisch darstellt und ausformuliert, ist Hölderlin eigen und geht über Spinoza hinaus, auch wenn zwischen Spinozas dritter Erkenntnisart, der „scientia intuitiva“, und dem ästhetischen Akt Hölderlins eine operative Äquivalenz besteht, insofern in beiden Akten das Allgemeine sich als Einzelnes und als Individualität realisiert. ${ }^{8}$

\section{Das absolute Sprachkunstwerk: Die späten Hymnen als Selbst-Aussage des All-Einen}

Im dritten und letzten Teil seien die Konsequenzen der Ausdrucksphilosophie für Hölderlins künstlerische Form angedeutet.

a) Dazu einige Bemerkungen über das Verhältnis der endlichen Modi zueinander: Es gibt keine substantielle Einheit im Bereich der Modi. Es handelt sich im Bereich der Ausdehnung um Geschwindigkeitsverhältnisse oder um eine repräsentative ,Verdichtung' von Ideen im Bereich des Denkens. In einem Brief an Sinclair vom 24. 12. 1798 wird dieses Wechselverhältnis der Modi, deren relationale Konstitution und Verfassung, von Hölderlin auf folgende Weise charakterisiert: „Alles greift ineinander und leidet, so wie es tätig ist.“ Dies entspricht der Konzeption des Spinoza, dass es zu jedem Modus im Universum einen umfassenderen gibt, durch den er begrenzt wird und durch den er insofern leidet.

b) Die relationalen Verhältnisse im Bereich der Modi, deren Konstitution durch Geschwindigkeitsdifferenzen bzw. durch ,Verdichtung' der Repräsentationen in einer Idee, sind auch ausschlaggebend für Hölderlins Lehre vom Wechsel der Töne.

Denn die Töne sind als Modifikationen der Sphäre der Tönbarkeit aufzufassen. Sie sind Bestimmtheiten, Modifikationen der phonetischen Klangwelt sowie Modi-fikationen der Sprachlautwelt und der damit verbundenen semantischen Gehalte. In der Sphäre der Töne sind Dissonanzen und Konsonanzen möglich. Gemäß einer Stelle aus dem Hyperion:

Beständigkeit haben die Sterne gewählt, in stiller Lebensfülle wallen sie stets und kennen das Alter nicht. Wir stellen im Wechsel das Vollendete dar; in wandelnden Melodien teilen wir die großen Akkorde der Freude. Wie Harfenspieler um die Throne der Ältesten, leben

\footnotetext{
${ }^{7}$ Man vgl. dazu Hölderlins Brief vom 24. 2. 1796 an Niethammer: „In den philosophischen Briefen will ich das Prinzip finden, das mir die Trennungen, in denen wir denken und existieren, erklärt, das aber auch vermögend ist, den Widerstreit zwischen dem Subjekt und dem Objekt, zwischen unserem Selbst und der Welt, ja auch zwischen Vernunft und Offenbarung - theoretisch in intellektualer Anschauung, ohne daß unsere praktische Vernunft zu Hilfe kommen müßte. Wir bedürfen dafür ästhetischen Sinn, und ich werde meine philosophischen Briefe ,Neue Briefe über die ästhetische Erziehung des Menschen` nennen." (6,1,Nr. 117)

${ }^{8}$ Vgl. dazu Wegenast (1990), S. 127 f., wo sie in Bezug auf die Fassung des Hyperion von 1799 schreibt: „Die dichterische Leistung besteht demzufolge wesentlich in der Erkenntnis dieses Begründungszusammenhangs, die Hölderlin in unmissverständlicher Anlehnung an die scientia intuitiva Spinozas als Fähigkeit zu schnellem Begriff kennzeichnet (...)
} 
wir, selbst göttlich, um die stillen Götter der Welt, mit dem flüchtigen Lebenslichte mildern wir den seligen Ernst des Sonnengotts und der andern. (Hyperion, 3: 148)

Das bedeutet auch: Jeder Ton der Sphäre verlangt nach der Ergänzung durch die anderen Töne. Damit steckt in jedem Ton eine Dynamik, der auf die Dynamik der anderen Töne verweist. Durch den permutierenden Tonwechsel wird ein selbstreferentielles System im Gedicht und als Gedicht erzeugt.

c) Um die späten Hymnen insgesamt richtig verstehen zu können, muss man sich klarmachen, dass Hölderlin eine absolute Poesie entwickelt, die selbstrefe-rentiell ist.

Das heißt: Das jeweilige Gedicht ist die Sache selbst, um die es geht. Hölderlins Dichtung spricht nicht über andere Ereignisse, Tugenden, Gefühle oder Ideale, sondern das einzelne Gedicht ist die poetische Konstruktion die Sache selbst, die im Akt des Lesens durch den Leser oder Hörer als absolutes Zeichen und damit als Mythe konstruiert wird.

Alle diejenigen geschichtlichen Ereignisse, die im Gedicht konstruiert werden, sind nur im Gedicht als diese Ereignisse wirklich, real. Sie entstehen als die so gedeuteten Ereignisse erst im Gedicht, und das Gedachte ist selbst die Wirklichkeit dieser Ereignisse.

Darum kann Hölderlin in seiner gnomisch-verdichteten Sprache die Selbst-referentialität des Gedichts im Gedicht Am Quell der Donau folgendermaßen aussprechen:

Jetzt aber endiget, seligweinend,

Wie eine Sage der Liebe

mir der Gesang

Und so auch ist er mir, mit Erröten, Erblassen

Von Anfang her gegangen.

Doch alles geht so. (Am Quell der Donau: 2,1: 129)

Der Gesang ist diese Sage der Liebe in doppeltem Sinn: Als genitivus objectivus und als genitivus subjectivus. Der Gesang ist Produkt und Resultat der Liebe und er ist zugleich diese Liebe selbst. Dies entspricht - kurz gesagt - formal der Selbstreferentialität des spinozanischen „amor Dei intellectualis“. Die Liebe zu Gott ist Gottes Liebe zu sich selbst.

Folgt man dem Gedanken, dass es Spinoza ist, der Hölderlin diese Brücke über die „Kantische Grenzlinie“ (Brief an Neuffer v. 10. 10. 1794) baut, indem das Sprachkunstwerk Vollzug und Realität des All-Einen ist, dann erscheint auch Hölderlins Gottesbegriff in den späten Hymnen in einem anderen Licht.

Der viel zitierte Anfang der Hymne Patmos lautet: „Nah ist / Und schwer zu fassen der Gott." Vor der Folie von Spinozas Ethik heißt das: Gott ist nah, weil ich sein Ausdruck bin, so dass in jedem meiner Denkakte bzw. meiner Körperbewegungen er sich als meine Identität erweist. Gott ist mir näher als mein eigenes Ich. Oder wie Spinoza sagt: Von Gott gibt es die höchste Gewißheit („summa certitudo“; E 1P11S; SO 2,54). Zugleich ist er schwer zu fassen, denn dazu muss ich erst mich selbst fassen und mein eigenes Wesen erkennen.

So gelesen, führen Kerngedanken Hölderlins zum Abschlußgedanken von Spinozas Ethik: Sich als Ausdruck der Substanz und insofern sich als ewig zu wissen. Dieser Gedanke, diese „Klarheit“, wie es in der Vorstufe zu seiner späteren Fassung der Patmos-Hymne heißt, ist das Rettende, das in der Gefahr wächst.

Nur Spinozas Konzeption des Ausdrucks konnte im Jahr 1800 die intellektuell-ästhetischen Probleme, mit denen sich Hölderlin auf dem Weg zu einer neuen Mythologie der Vernunft konfrontiert sah, auf moderne Weise lösen. 


\section{Literaturverzeichnis}

Friedrich Heinrich Jacobi’s Werke. 6 Bde., Leipzig 1812-1825, hrsg. v. Friedrich Roth u. Friedrich Köppern, Reprographischer Nachdruck, 2. Aufl., Darmstadt 1976.

Heinse, Wilhelm: Ardinghello und die glückseligen Inseln. Kritische Studienausgabe, hrsg. v. Max L. Baeumer, Stuttgart 1978.

Hölderlin, Friedrich: Sämtliche Werke. Historisch-kritische Ausgabe, hrsg. v. Friedrich Beißner. Stuttgart 1943 ff. Spinoza, Baruch de: Opera. Im Auftrag der Heidelberger Akademie der Wissenschaften hrsg. v. Carl Gebhardt, Heidelberg 1972 (Bd. 5 1989).

\section{Literatur}

Büttner, Stefan: Gott und Raum. Spinozas Konzeption der Ausdehnung und Körperwelt. Würzburg: Königshausen \& Neumann, 2009.

Büttner, Stefan: Natur. Ein Grundwort Hölderlins,„Natur - ein Grundwort Hölderlins“. In: Hölderlin-Jahrbuch 1988/1989, 223-247.

Reisinger, Peter: „Hölderlin zwischen Fichte und Spinoza. Der Weg zu Hegel“. In: Bachmeier, Helmut u. Rentsch, Thomas (Hrsg.): Poetische Autonomie? Zur Wechselwirkung von Dichtung und Philosophie in der Epoche Goethes und Hölderlins. Stuttgart: Klett-Cotta, 1987: 15-69.

Unveränderter Wiederabdruck in: Friedrich Hölderlin. Neue Wege der Forschung, hrsg. von Thomas Roberg, Darmstadt 2003, 227-252.

Wegenast, Margarethe: Hölderlins Spinoza-Rezeption und ihre Bedeutung für die Konzeption des Hyperion. Tübingen: Niemeyer, 1990. 